\title{
Preparation and characterization of tetrandrine- phospholipid complex loaded lipid nanocapsules as potential oral carriers
}

This article was published in the following Dove Press journal:

International Journal of Nanomedicine

30 October 2013

Number of times this article has been viewed

\section{Yi-qing Zhao \\ Li-ping Wang \\ Chao Ma \\ Kun Zhao \\ Ying Liu \\ Nian-ping Feng}

School of Pharmacy, Shanghai University of Traditional Chinese Medicine, Shanghai, People's Republic of China
Correspondence: Ying Liu

Department of Pharmaceutics, School of Pharmacy, Shanghai University of Traditional Chinese Medicine, I 200 Cailun Road, Zhangiiang Hi-Tech Park, Pudong New District, Shanghai 20I203, People's Republic of China

$\mathrm{Tel}+862151322210$

Fax +86 2I 5132 2198

Email debbyly@163.com

Nian-ping Feng

Department of Pharmaceutics, School

of Pharmacy, Shanghai University of Traditional Chinese Medicine,

I 200 Cailun Road, Zhangjiang Hi-Tech

Park, Pudong New District, Shanghai

201203, People's Republic of China

$\mathrm{Tel}+862151322198$

Fax +86 2I 5132 2198

Email npfeng@hotmail.com
Background: Tetrandrine is an active constituent that is extracted from the root tuber of the Chinese herb Stephania tetrandra S. Moore. It has shown various pharmacological effects, such as antitumor activity, multidrug resistance reversal, and hepatic fibrosis resistance. In clinical applications, it has been used to treat hypertension, pneumosilicosis, and lung cancer. However, the poor water solubility of tetrandrine has limited its application. In this study, a newly emerging oral drug carrier of phospholipid complex loaded lipid nanocapsules was developed to improve the oral bioavailability of tetrandrine.

Methods: The phospholipid complex was prepared with the solvent-evaporation method to enhance the liposolubility of tetrandrine. The formation of the phospholipid complex was confirmed with a solubility study, infrared spectroscopy, and a differential scanning calorimetry (DSC) analysis. The tetrandrine-phospholipid complex loaded lipid nanocapsules (TPC-LNCs) were prepared using the phase inversion method. Lyophilization was performed with mannitol $(10 \%)$ as a cryoprotectant. TPC-LNCs were characterized according to their particle size, zeta potential, encapsulation efficiency, morphology by transmission electron microscopy, and crystallinity by DSC. In addition, the in vitro release of tetrandrine from TPC-LNCs was examined to potentially illustrate the in vivo release behavior. The in vivo bioavailability of TPC-LNCs was studied and compared to tetrandrine tablets in rats.

Results: The liposolubility of tetrandrine in $n$-octanol improved from $8.34 \mu \mathrm{g} / \mathrm{mL}$ to $35.64 \mu \mathrm{g} / \mathrm{mL}$ in the tetrandrine-phospholipid complex. The prepared TPC-LNCs were sphericalshaped particles with a small size of $40 \mathrm{~nm}$ and a high encapsulation efficiency of $93.9 \%$. DSC measurements revealed that the crystalline state was less ordered in lipid nanocapsules. The in vitro release study demonstrated a fast release of approximately $25 \%$ in the first 1 hour, which was followed by a sustained release of $70 \%$ over 12 hours. The relative bioavailability of TPCLNCs compared to that of tablets was $208 \%$, indicating a significant improvement in the oral absorption of tetrandrine.

Conclusion: The TPC-LNCs system developed in this study is a promising carrier that improves the oral bioavailability of tetrandrine in rats. The phospholipid complex loaded lipid nanocapsules have great potential for use as an oral drug delivery system for moderately lipophilic drugs that are encapsulated in the lipid nanocapsules.

Keywords: tetrandrine, phospholipid complex, lipid nanocapsules, oral bioavailability, enhanced liposolubility

\section{Introduction}

Tetrandrine (Figure 1), which is a bisbenzylisoquinoline alkaloid, is the major active constituent that is extracted from the root tuber of the Chinese herb Stephania tetrandra S. Moore. Tetrandrine has been found to be clinically effective for the treatment of 


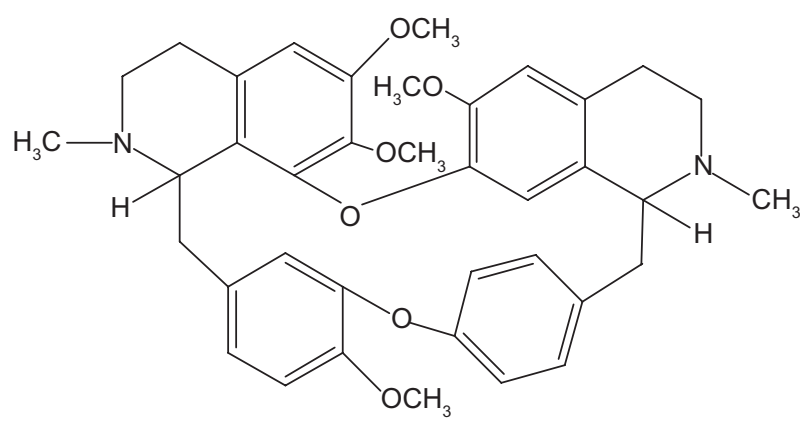

Figure I Chemical structure of tetrandrine.

inflammation, hypertension, pneumosilicosis, and lung cancer. ${ }^{1}$ In addition, tetrandrine has exhibited other promising pharmacological effects, including antitumor activity, ${ }^{2}$ reversing multidrug resistance, ${ }^{3}$ and hepatic fibrosis resistance. ${ }^{4}$ It is also a nonselective calcium channel blocker and calmodulin antagonist. ${ }^{5}$ Although it has potentially considerable value clinically, some problems have arisen that have discouraged tetrandrine clinical application. In general, a large dose (6 to 15 tablets per day) is required for the current commercially available tablets. In addition, its poor solubility in water contributes to its low and variable oral bioavailability. In recent years, some investigations have been reported that have focused on improving the bioavailability of tetrandrine, such as emulsions, ${ }^{6}$ solid lipid nanoparticles, ${ }^{7}$ liposomes, ${ }^{8}$ and microspheres. ${ }^{9}$ Most of the reported preparations have been administered by injection. Alternative novel oral drug delivery systems are of great value in that they contribute to the greatest degree of patient compliance, enhance bioavailability, and, thus, result in better therapeutic effects.

In recent years, lipid-based nanocarriers have drawn attention for their potential as drug delivery systems for the oral delivery of drugs with poor water solubility. ${ }^{10}$ The advantages of lipid-based nanocarriers include not only the enhanced bioavailability of drugs with poor water solubility, but also improvements in the uptake and release of drugs and protection against degradation. ${ }^{11}$ Various lipid-based nanocarriers, such as liposomes, lipid micelles, solid lipid nanoparticles, nanoemulsions, lipid nanocapsules (LNCs), and nanosuspensions, have been investigated. Among these, LNCs offer a promising pathway for the oral delivery of drugs with poor water solubility. ${ }^{12}$ LNCs were first introduced by Heurtault et al who used the phase inversion technique. ${ }^{13}$ LNCs, which are composed of medium-chain triglycerides and polymers/ surfactant, are characterized by a hybrid structure that is between polymer nanoparticles and liposomes. Their size ranges from $25 \mathrm{~nm}$ to $100 \mathrm{~nm}$. Except for their advantages that are similar to other lipid carriers, the specific features or advantages of LNCs over other lipid nanocarriers include the following: they possess a liquid core and a tensioactive and cohesive rigid membrane at the body temperature of $37^{\circ} \mathrm{C} ; 1^{14}$ compared to liposomes, they can be prepared using a solvent free, soft-energy procedure that utilizes the phase inversion principle; $;^{15}$ and less solubilizer agents are used in their formulation compared to emulsions or microemulsions. ${ }^{15}$ LNCs have been shown to enhance the oral bioavailability of the poorly water-soluble drug paclitaxel. ${ }^{16}$ The proposed mechanisms for this include improved solubilization, increased lymphatic absorption, P-glycoprotein inhibition, and multidrug resistance reversal.

In recent years, LNCs have been investigated for the delivery of several poorly water-soluble drugs, such as paclitaxel and docetaxel, and they exhibited a high encapsulation efficiency of over $90 \% .{ }^{16-18}$ However, our results have shown that tetrandrine loaded LNCs have a low encapsulation efficiency of less than $70 \%$, which may be due to the moderate lipophilicity of tetrandrine $(\log \mathrm{P} 2.5[\mathrm{pH} 7.0])$. Encapsulation efficiency is an important parameter of nanocarrier formulation with respect to clinical application and drug cost. Low encapsulation efficiency may result in poor therapeutic effects and the requirement of a higher dose of the drug. In addition, it has been identified that there are two critical quality attributes in the development of lipid-based drug delivery systems: one is the drug solubility within the lipidic system, and the other is the physiological processing of lipid carriers to achieve the desirable drug absorption. ${ }^{19}$ The use of more surfactants may facilitate the drug being dissolved or encapsulated in lipid carriers by solubilization. However, cytotoxicity or irritation to the gastrointestinal tract mucus membranes may occur due to an excess amount of surfactants..$^{20}$ Therefore, in order to obtain qualified LNCs, it is necessary to seek an efficient and safe strategy to improve the affinity between tetrandrine and LNCs and to enhance tetrandrine encapsulation efficiency in LNCs.

Previous investigations have shown that the formation of the drug-phospholipid complex enhanced the lipophilicity of the drugs. ${ }^{21,30}$ Therefore, it may facilitate the encapsulation of drug molecules into hydrophobic nanocarriers and result in higher encapsulation efficiency and better therapeutic effects. In addition, although a drug-phospholipid complex has the capacity of improving drug absorption and bioavailability, ${ }^{22,23}$ the low water solubility of the complex has limited its application. ${ }^{24}$ Therefore, loading or combining drug-phospholipid complex with nanocarriers has been introduced in an effort to mitigate some limitations associated with the complex and nanocarriers. Drug-phospholipid 
complex has been successfully used in several drug delivery systems, such as poly(lactic-co-glycolic acid) nanoparticles, ${ }^{25}$ self-nanoemulsified drug delivery systems, ${ }^{26}$ poly(hydroxybutyrate-co-hydroxyhexanoate) nanoparticles, ${ }^{27}$ and mixed micelles. ${ }^{24}$ To the best of our knowledge, no studies of LNCs that are loaded with a drug-phospholipid complex have been reported to date.

The objective of this study was to develop tetrandrinephospholipid complex loaded LNCs (TPC-LNCs) as a carrier in order to facilitate the incorporation of tetrandrine into LNCs and improve the oral bioavailability of tetrandrine. The preparations were evaluated in vitro with transmission electron microscopy (TEM) observations, differential scanning calorimetry (DSC), and an in vitro release study. Furthermore, an in vivo bioavailability study after oral administration was performed in order to investigate its pharmacokinetic behavior and bioavailability enhancement.

\section{Materials and methods Materials}

Tetrandrine (purity $>98 \%$ ) was purchased from Jiangxi Herbfine Hi-Tech Co, Ltd (Nanchang, People's Republic of China). Labrafil ${ }^{\circledR}$ M1944CS (Oleoyl macrogol-6 glycerides) was a gift from Gattefossé (Saint Priest, France). Lipoid S75 (soybean lecithin at 69\% of phosphatidylcholine) from Lipoid Gmbh (Ludwigshafen, Germany) was provided by Shanghai Toshisun Biology and Technology Co, Ltd (Shanghai, People's Republic of China). Solutol ${ }^{\mathbb{R}}$ HS15 (PEG660-12-hydroxystearate) was obtained from BASF (Ludwigshafen, Germany). $\mathrm{NaCl}$ was obtained from Sinopharm Chemical Reagent Co, Ltd (Shanghai, People's Republic of China). Deionized water and purified water was obtained using a Millipore ${ }^{\circledR}$ Simplicity System (Millipore, Billerica, MA, USA). All other solvents were of analytical reagent grade or high-performance liquid chromatography (HPLC) grade.

\section{Animal models}

Male Sprague-Dawley rats (clean grade, $250 \pm 20 \mathrm{~g}$ ) were used in the in vivo bioavailability study. The protocol for the animal studies was approved by the institutional animal ethical committee of the Shanghai University of Traditional Chinese Medicine. The rats were bred and maintained for at least 1 week as an acclimatization period before the study under the standard conditions at the Laboratory Animal Center of the Shanghai University of Traditional Chinese Medicine.

\section{Preparation of the tetrandrine- phospholipid complex}

The tetrandrine-phospholipid complex (TPC) was prepared with the solvent-evaporation method. Briefly, tetrandrine and phospholipids at a molar ratio of 1:1 were dissolved in tetrahydrofuran under magnetic stirring at room temperature. The obtained mixture was continuously stirred for 24 hours. Then, tetrahydrofuran was removed with a rotary evaporator under reduced pressure. The sample was vacuum dried for 48 hours. The resultant complex was firmly sealed for further use.

\section{TPC characterization}

\section{Solubility determination}

Excess TPC was added to distilled water and $n$-octanol. The mixture was gently stirred for 24 hours at $25^{\circ} \mathrm{C}$. The samples were centrifuged at $2,500 \times g$ for 20 minutes. The supernatant was filtered with a $0.45 \mu \mathrm{m}$ filter. The contents of tetrandrine were analyzed by HPLC. The HPLC analysis was performed with an Agilent HPLC system (HP 1200, Agilent Technologies, Inc, Santa Clara, CA, USA). The experiment consisted of an octadecylsilyl column (Platisil ${ }^{\mathrm{TM}} \mathrm{C} 18$ [Dikma Technologies, Lake Forest, CA, USA], $250 \mathrm{~mm} \times 4.6 \mathrm{~mm}$, $5 \mu \mathrm{m})$. The mobile phase consisted of acetonitrile/water/ triethanolamine (80:20:0.01, volume $[\mathrm{v}] / \mathrm{v} / \mathrm{v})$ with a flow rate of $1.0 \mathrm{~mL} /$ minute. The column temperature was $30^{\circ} \mathrm{C}$, and the detector was $282 \mathrm{~nm}$. The solubility of tetrandrine was measured with the same method and compared with that of TPC in order to determine the change in solubility that was caused by complexation.

\section{DSC}

The DSC analysis was performed with a NETZSCH DSC 204 apparatus (NETZSCH Gerätebau GmbH, Selb, Germany). A scan rate of $10^{\circ} \mathrm{C} /$ minute was employed. The temperature range was $30^{\circ} \mathrm{C}-250^{\circ} \mathrm{C}$. The analysis was performed under a nitrogen purge. An empty aluminum pan was used as a reference.

\section{Infrared spectroscopy}

Infrared (IR) spectroscopic spectral analyses of tetrandrine, phospholipid, TPC, and the physical mixture of tetrandrine with phospholipid were performed with a Fourier transform infrared spectrometer (AVATAR 330, Thermo Scientific, Waltham, MA, USA) in order to confirm the formation of the TPC. The measurement was conducted by the conventional transmission mode with a $\mathrm{KBr}$ pellet. A proper amount of each material $(1 \%$, weight $[\mathrm{w}] / \mathrm{w}$, with respect to $\mathrm{KBr})$ was ground and mixed with dry $\mathrm{KBr}$ powder, which was followed 
by compressing it into a $\mathrm{KBr}$ disc. The resultant pellet was scanned in the range of $400-4,000 \mathrm{~cm}^{-1}$ with a $4 \mathrm{~cm}^{-1}$ resolution. The results were recorded and analyzed with OMNIC (ver 7.1) IR solution software (Thermo Scientific).

\section{Preparation of TPC-LNCs}

LNCs were prepared based on slight modification of the phase inversion process that has been previously described. ${ }^{13}$ Briefly, TPC and phospholipids were totally dissolved in Labrafil M1944CS at $70^{\circ} \mathrm{C}$ by gentle agitation. The prepared oil phase was mixed with the aqueous solution that contained Solutol HS15, NaCl, and distilled water. The resultant mixture was heated under magnetic stirring from room temperature to $90^{\circ} \mathrm{C}$. Three temperature cycles from $40^{\circ} \mathrm{C}$ to $90^{\circ} \mathrm{C}$ were performed at the temperature rate of $4^{\circ} \mathrm{C} / \mathrm{min}$ in order to reach the phase inversion process. Then, the mixture was cooled to $55^{\circ} \mathrm{C}$, which was followed by dilution with $0^{\circ} \mathrm{C}$ deionized water and continuous stirring for $10 \mathrm{~min}$ at room temperature.

\section{Preparation of lyophilized TPC-LNCs}

TPC-LNCs were lyophilized using the following cryoprotectants: lactose, sucrose, glucose, and mannitol. In order to screen the cryoprotectants, the cryoprotectant was dissolved in the TPC-LNC dispersion to the predetermined concentration of $2 \%, 5 \%$, or $10 \%(\mathrm{w} / \mathrm{v})$. The obtained mixture was frozen at $-70^{\circ} \mathrm{C}$ for 5 hours. Then, freeze-drying was conducted with a freeze dryer (Christ Epsilon 2-4 LSC, Martin Christ Gefriertrocknungsanlagen $\mathrm{GmbH}$, Osterode am Harz, Germany) for 24 hours until complete solidification. The lyophilized LNCs were evaluated and compared to those before lyophilization by appearance, reconstitution testing, droplet size, and polydispersity measurement. For the reconstitution test, a proper amount of distilled water was added to the dried sample to its initial volume, and the redispersion rate and extent was recorded. Droplet size and polydispersity measurements were conducted according to the following described method.

\section{Characterization of TPC-LNCs Determination of encapsulation efficiency}

The encapsulation efficiency of tetrandrine in LNCs was determined with slight modification of a previously reported method. ${ }^{16}$ Briefly, the preparations were filtered with a $0.45 \mu \mathrm{m}$ filter in order to remove the tetrandrine precipitation. Then, the filtrate was dissolved with a methanol/tetrahydrofuran $(96: 4, \mathrm{v} / \mathrm{v})$ solution, which was followed by filtration through a $0.2-\mu \mathrm{m}$ Minisart ${ }^{\circledR}$ high-flow filter (Sartorius AG,
Göttingen, Germany) in order to remove the residuals in the LNCs. A $20 \mu \mathrm{L}$ aliquot of the obtained filtrate was used in the tetrandrine analysis by HPLC. The encapsulation efficiency was calculated as follows:

$$
\text { Encapsulation efficiency }(\%)=\left(\mathrm{W}_{1} / \mathrm{W}_{2}\right) \times 100 \%
$$

where $\mathrm{W}_{1}$ represents the drug amount that was encapsulated in the LNC postformulation, and $\mathrm{W}_{2}$ represents the drug amount that was added in the formulation process.

\section{Determination of droplet size and zeta potential}

After the preparations were diluted with distilled water $(1: 100 \mathrm{v} / \mathrm{v})$, the droplet size and zeta potential of the sample were determined with a Nano ZS90 Zetasizer (Malvern Instruments Ltd, Malvern, UK). Each sample was analyzed in triplicate.

\section{TEM observation}

The morphology of TPC-LNCs was observed by TEM (JEM-1230; JEOL Ltd, Tokyo, Japan). TPC-LNCs were diluted with distilled water and gently mixed. Then, a drop of mixture was placed on copper grids, which was followed by drawing off the excess with filter paper. Subsequently, it was stained in $1 \%$ phosphotungstic acid solution for 30 seconds. The specimen was air-dried for approximately 2 hours before observation.

\section{DSC analysis}

The DSC analysis of the TPC-LNCs was performed with the same method that was described for TPC characterization.

\section{In vitro release}

Tetrandrine release from TPC-LNCs was conducted with a dialysis method in $100 \mathrm{~mL}$ of phosphate-buffered saline $(\mathrm{pH}$ 6.8 ) containing $0.5 \%$ Tween 80 at $37^{\circ} \mathrm{C} .{ }^{28}$ Two milliliters of the sample was placed in a dialysis bag (molecular weight cutoff, 14,000). The bag was then firmly tied and immersed in medium in a shaker bath (100 strokes/min). At a defined time interval, $200 \mu \mathrm{L}$ of the sample was withdrawn and replaced with the same volume of fresh medium. The tetrandrine concentration was measured by HPLC. Tetrandrine release of free drugs and TPC were performed using the same method, respectively.

\section{In vivo bioavailability}

The bioavailability of the TPC-LNCs was compared to that of the tetrandrine tablets. The rats were fasted for 12 hours before dosing. Water was available throughout the study period. 
The rats were divided into two groups with five animals in each group. One group received TPC-LNCs and one group received tetrandrine tablets by oral gavage (dose of $2.4 \mathrm{mg} / \mathrm{kg}$ ). The tablets were crushed gently, and then diluted using distilled water before administration to rats. Approximately $0.5 \mathrm{~mL}$ of blood was taken from the retroorbital plexus and placed into heparinized tubes before administration and at 10 minutes, 30 minutes, 45 minutes, 1 hour, 1.6 hours, 5 hours, 8 hours, 12 hours, and 24 hours after dosing. The plasma was separated by centrifugation at $2,000 \times g$ for 10 minutes. Plasma was stored at $-20^{\circ} \mathrm{C}$ until the analysis, as described below.

A total of $100 \mu \mathrm{L}$ of plasma was transferred into a tube and mixed with an equal volume of acetonitrile. Samples were vortexed for about 1 minute. After centrifugation at $13,201 \times g$ for 15 minutes at $4^{\circ} \mathrm{C}$, the supernatant was taken for HPLC analysis. The HPLC assay of the tetrandrine plasma concentrations was performed with an Agilent 1200 HPLC system (Agilent Technologies). The conditions were as follows: a column flow rate of $1.0 \mathrm{~mL} /$ minute, a mobile phase of acetonitrile/water/triethanolamine (80:20:0.01, v/v/v), and a detector wavelength of $282 \mathrm{~nm}$. The linear calibration curve was obtained between $0.02 \mu \mathrm{g} / \mathrm{mL}$ and $8.00 \mu \mathrm{g} / \mathrm{mL}$ with a correlation coefficient of 0.9995 .

\section{Data analysis}

A pharmacokinetic analysis was performed on the data of the tetrandrine plasma concentration versus time with DAS 2.0 software (Mathematical Pharmacology Professional Committee of China, Shanghai, People's Republic of China). The main pharmacokinetic parameters, including peak concentration $\left(\mathrm{C}_{\max }\right)$, peak time $\left(\mathrm{T}_{\max }\right)$, and the area under the curve (AUC), were obtained. The relative bioavailability $(F)$ of the LNCs to the tablets was calculated using the following equation:

$$
F=\frac{\mathrm{AUC}_{\text {test }}}{\mathrm{AUC}_{\text {reference }}} \times 100 \%
$$

The results were expressed as the mean \pm standard deviation. The statistical analysis was conducted with a oneway analysis of variance (ANOVA) in order to determine the significance of the differences. A $P$-value less than 0.05 was considered statistically significant.

\section{Results and discussion TPC: preparation, solubility, and characterization}

In recent years, LNCs have been reported to be a promising nanocarrier for the delivery of poorly water-soluble drugs. ${ }^{12}$
In our preliminary study, tetrandrine loaded LNCs were first designed and formulated. The formulation composition and results of the characterization are shown in Table 1.

It was clear that tetrandrine loaded LNCs possessed a qualified droplet size and polydispersibility. However, the encapsulation efficiency of tetrandrine in LNCs was lower than $70 \%$. Compared to the high encapsulation rate of paclitaxel or docetaxel in LNCs, ${ }^{16,17}$ the low encapsulation efficiency in this study may have been due to the moderate lipophilicity of tetrandrine $(\log \mathrm{P} 2.5[\mathrm{pH} 7.0])$. As described above, the formation of a drug-phospholipid complex has proved to be an efficient and safe strategy that improves the lipophilicity of a drug. In this study, TPC was prepared in order to improve the affinity between tetrandrine and LNCs and to obtain a higher encapsulation efficiency. The solubility of TPC and tetrandrine in water and $n$-octanol is presented in Table 2.

Compared to tetrandrine, TPC showed a much higher solubility both in water and in $n$-octanol. The solubility in $n$-octanol increased from $8.34 \mu \mathrm{g} / \mathrm{mL}$ to $35.64 \mu \mathrm{g} / \mathrm{mL}$, indicating an improvement in lipophilicity. The enhanced solubility in the complex may have been due to the solubilization of the drug and the amorphous features of the complex, ${ }^{29}$ which was shown by the DSC analysis in this study, and the complex may exhibit higher bioavailability in vivo.

DSC measurements were used to obtain distinct information on the polymorphism and crystallinity of the interaction between the drug and phospholipids from DSC thermograms that provide information, such as the elimination of endothermic peaks, the appearance of new peaks, and changes in peak shape and onset, peak temperature, or enthalpy. ${ }^{30}$ In this study, a DSC analysis was performed in order to study the association of tetrandrine with phospholipids. Figure 2 presents the DSC thermograms of tetrandrine, the phospholipids, the physical mixture of tetrandrine and phospholipids, and TPC. Tetrandrine showed 1 sharp peak at $225.7^{\circ} \mathrm{C}$. The thermogram of the phospholipids exhibited double peaks. The front gentle peak was from the thermal

Table I Results of measurements of droplet size, polydispersity, and encapsulation efficiency $(n=3)$

\begin{tabular}{llll}
\hline Formulation & $\begin{array}{l}\text { Droplet } \\
\text { size }(\mathbf{n m})\end{array}$ & Polydispersity & $\begin{array}{l}\text { Encapsulation } \\
\text { efficiency (\%) }\end{array}$ \\
\hline $\begin{array}{l}\text { Tetrandrine } \\
\text { loaded LNCs }\end{array}$ & $30.75 \pm 1.2$ & $0.083 \pm 0.05$ & $66.4 \pm 2.7$ \\
TPC-LNCs & $40.0 \pm 3.5$ & $0.155 \pm 0.08$ & $93.9 \pm 1.5$ \\
\hline
\end{tabular}

Note: Values are mean \pm standard deviation.

Abbreviations: LNCs, lipid nanocapsules; TPC, tetrandrine-phospholipid complex; TPC-LNC, TPC loaded LNCs. 
Table 2 Solubility of tetrandrine and TPC in water and $n$-octanol at $25^{\circ} \mathrm{C}(\mathrm{n}=3)$

\begin{tabular}{llc}
\hline Sample & $\begin{array}{l}\text { Solubility in } \\
\text { water }(\mu \mathrm{g} / \mathrm{mL})\end{array}$ & $\begin{array}{l}\text { Solubility in } \\
\text { n-octanol }(\mu \mathrm{g} / \mathrm{mL})\end{array}$ \\
\hline Tetrandrine & $0.46 \pm 0.1 \mathrm{I}$ & $8.34 \pm 0.52$ \\
TPC & $8.28 \pm 0.37$ & $35.64 \pm 0.46$ \\
\hline
\end{tabular}

Note: Values are mean \pm standard deviation.

Abbreviation: TPC, tetrandrine-phospholipid complex. motion of the polar group of the phospholipids, while the sharp peak occurred due to the phase transition from the gel phase to the liquid crystalline phase, which may be related to the melting of the hydrocarbon chain, isomerization, or the change of the crystal shape. ${ }^{31}$ The thermograms of the physical mixture showed three peaks at $192.6^{\circ} \mathrm{C}, 206.2^{\circ} \mathrm{C}$, and $221.4^{\circ} \mathrm{C}$ from the phospholipids (the former two peaks)

A

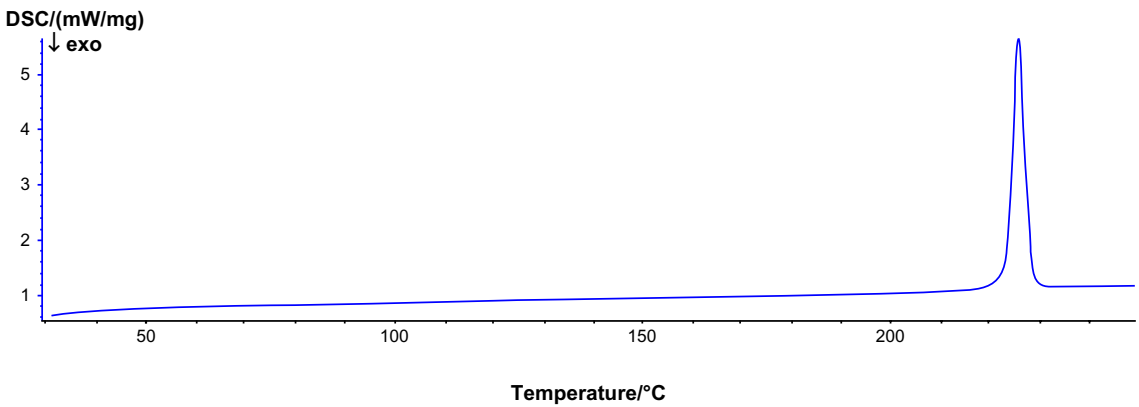

B $\mathrm{DSC} /(\mathrm{mW} / \mathrm{mg})$

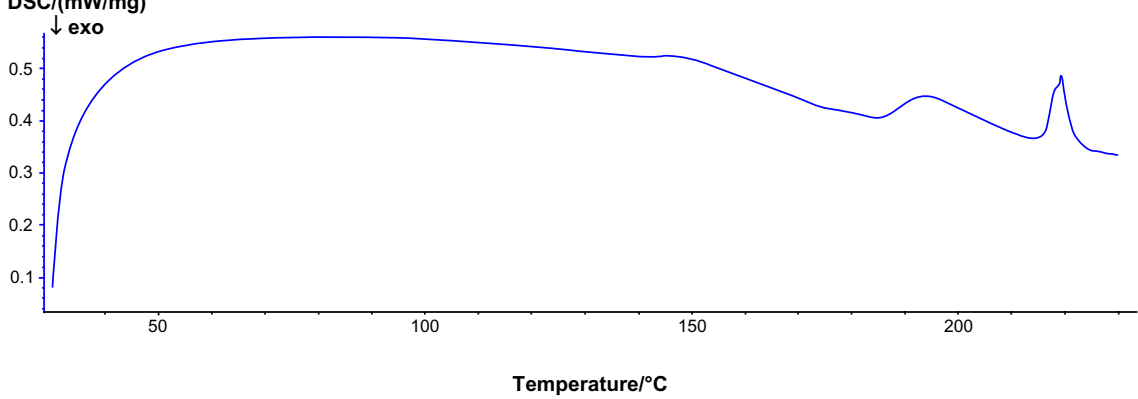

C $\mathrm{DSC} /(\mathrm{mW} / \mathrm{mg})$

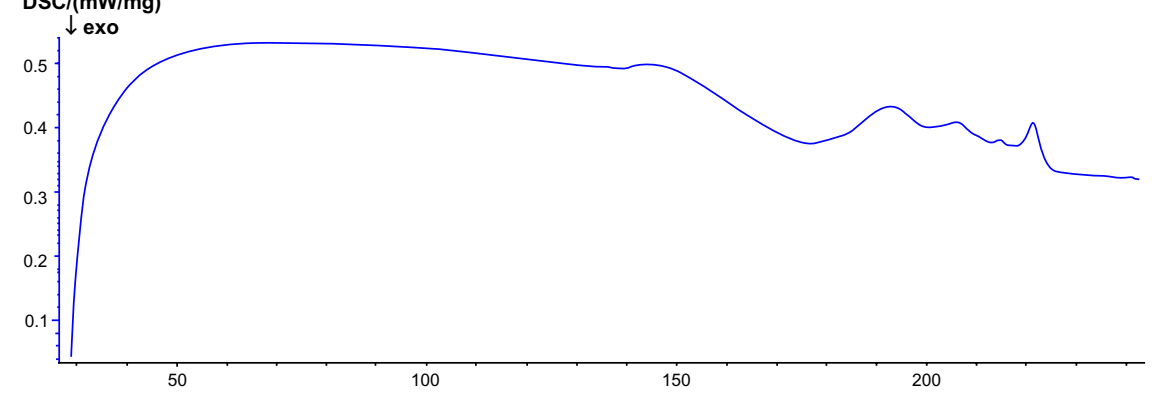

Temperature $/{ }^{\circ} \mathrm{C}$

D $\mathrm{DSC} /(\mathrm{mW} / \mathrm{mg})$

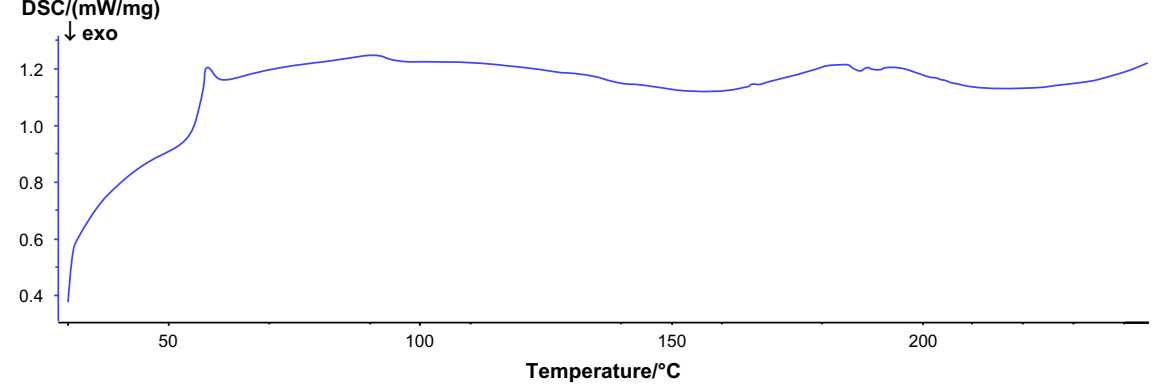

Figure 2 Differential scanning calorimetry spectra of $(\mathbf{A})$ tetrandrine, (B) phospholipids, $(\mathbf{C})$ the physical mixture of tetrandrine and phospholipids, and (D) the tetrandrinephospholipid complex.

Abbreviations: DSC, differential scanning calorimetry; mW, milliwatt; exo, exothermal. 
and tetrandrine. The phospholipids melted during the programmed heating, and a portion of the tetrandrine interacted with the phospholipids. Because of the limited heating time, some tetrandrine remained. These results are similar to those of a previous study. ${ }^{28}$ The TPC thermogram was clearly different from those of tetrandrine, the phospholipids, and the physical mixture. The peak of tetrandrine disappeared in the TPC thermogram, which indicated that tetrandrine may interact with phospholipids and form a complex. The reason for the disappearing peaks of tetrandrine was that the drug-phospholipid complex that formed were a special solid dispersion, which indicated that tetrandrine lost its crystallinity. ${ }^{32}$
The prepared TPC was characterized by IR spectroscopy. The IR spectra of tetrandrine, phospholipid, the TPC, and the physical mixture of tetrandrine and phospholipids are shown in Figure 3.

The IR spectra results showed that the pattern of the physical mixture of tetrandrine and phospholipids was similar to their overlaid patterns, and no new peaks appeared in the spectrum of the physical mixture. The physical mixture showed a broad peak, which was like that of phospholipid, at $3,409.51 \mathrm{~cm}^{-1}$, and sharp peaks of tetrandrine that were lower than $2,000 \mathrm{~cm}^{-1}$, indicating no chemical interaction between the phospholipids and tetrandrine. The complex spectrum was markedly different from that of the physical mixture and
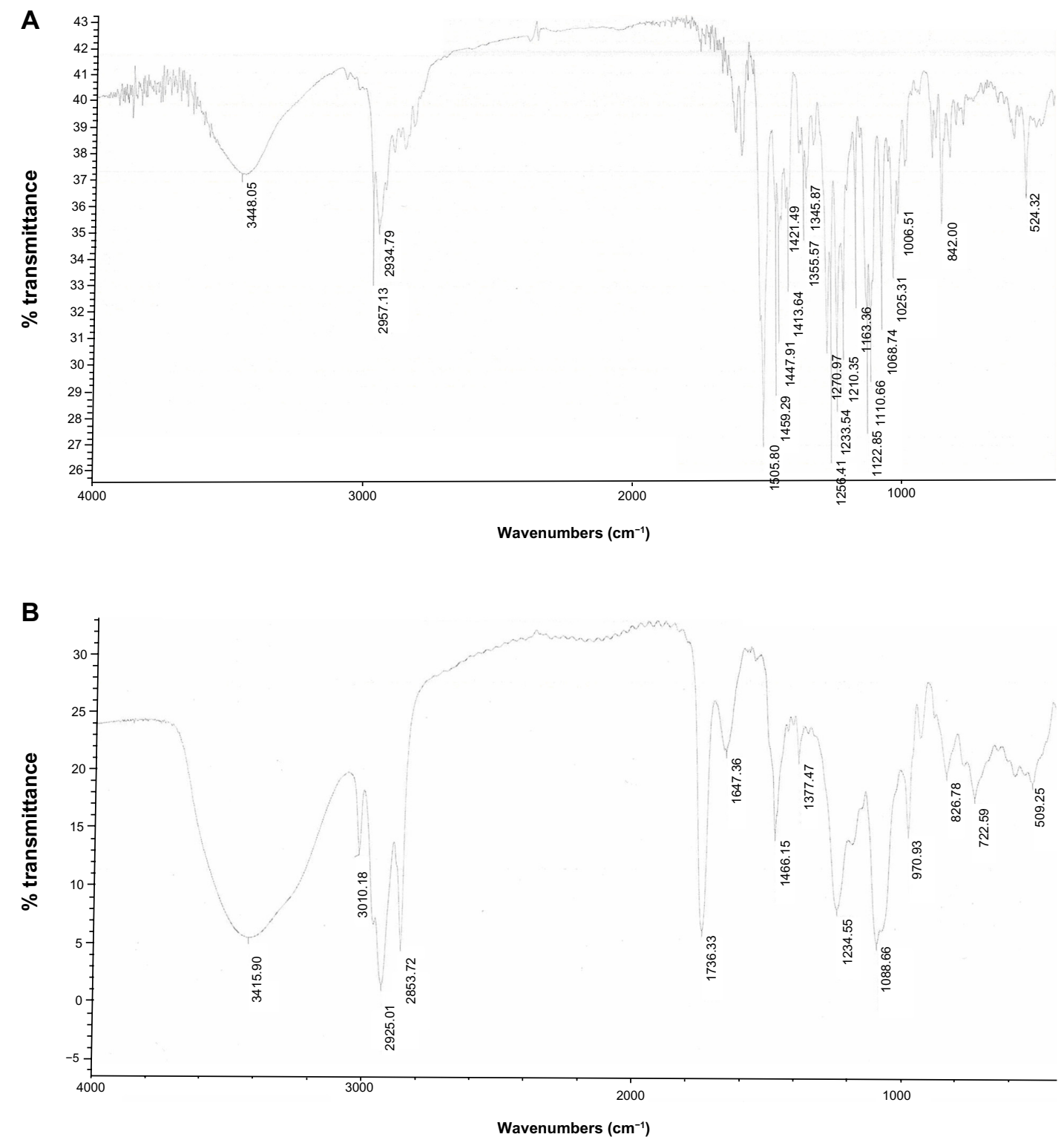

Figure 3 (Continued) 


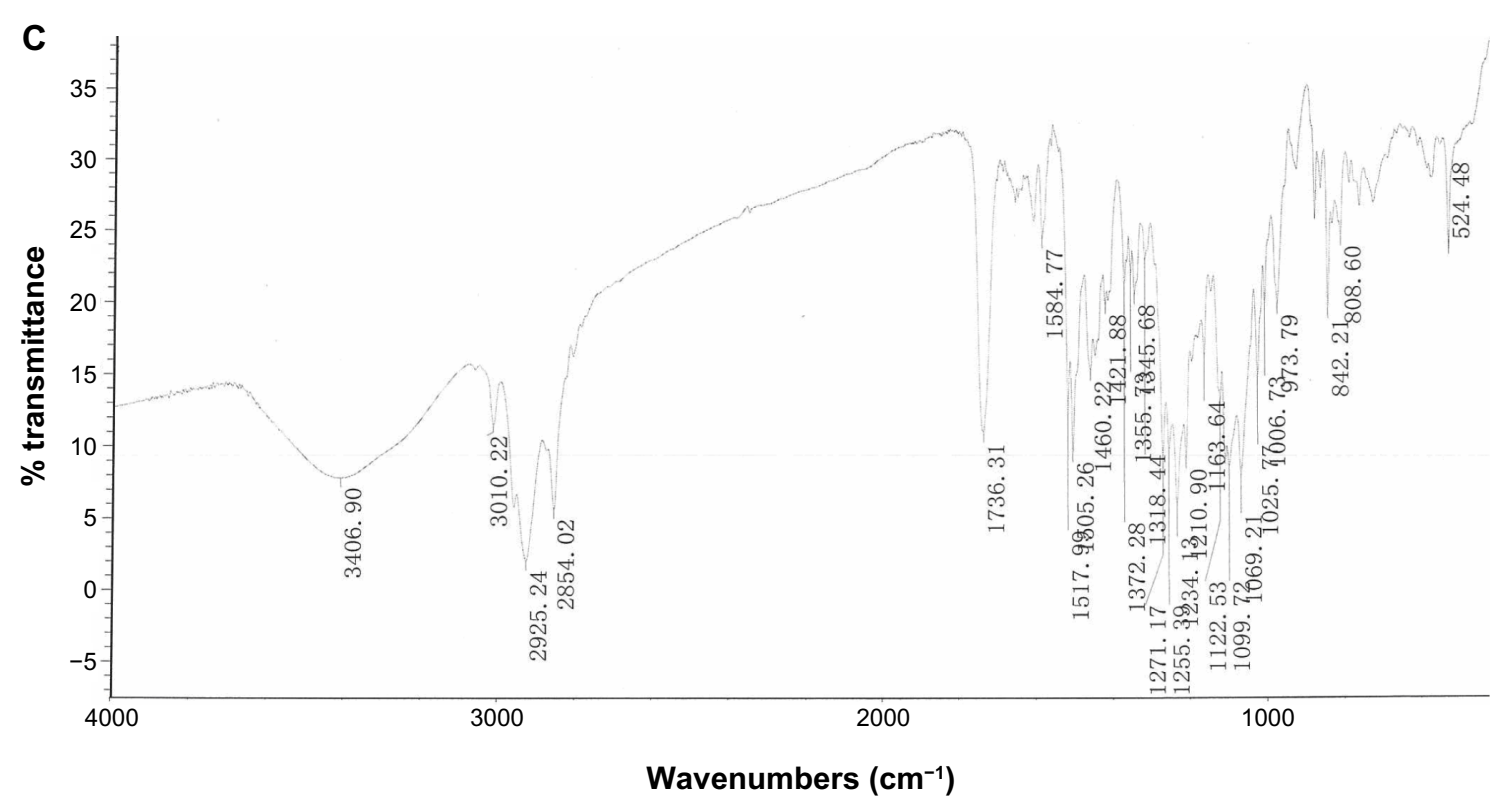

D

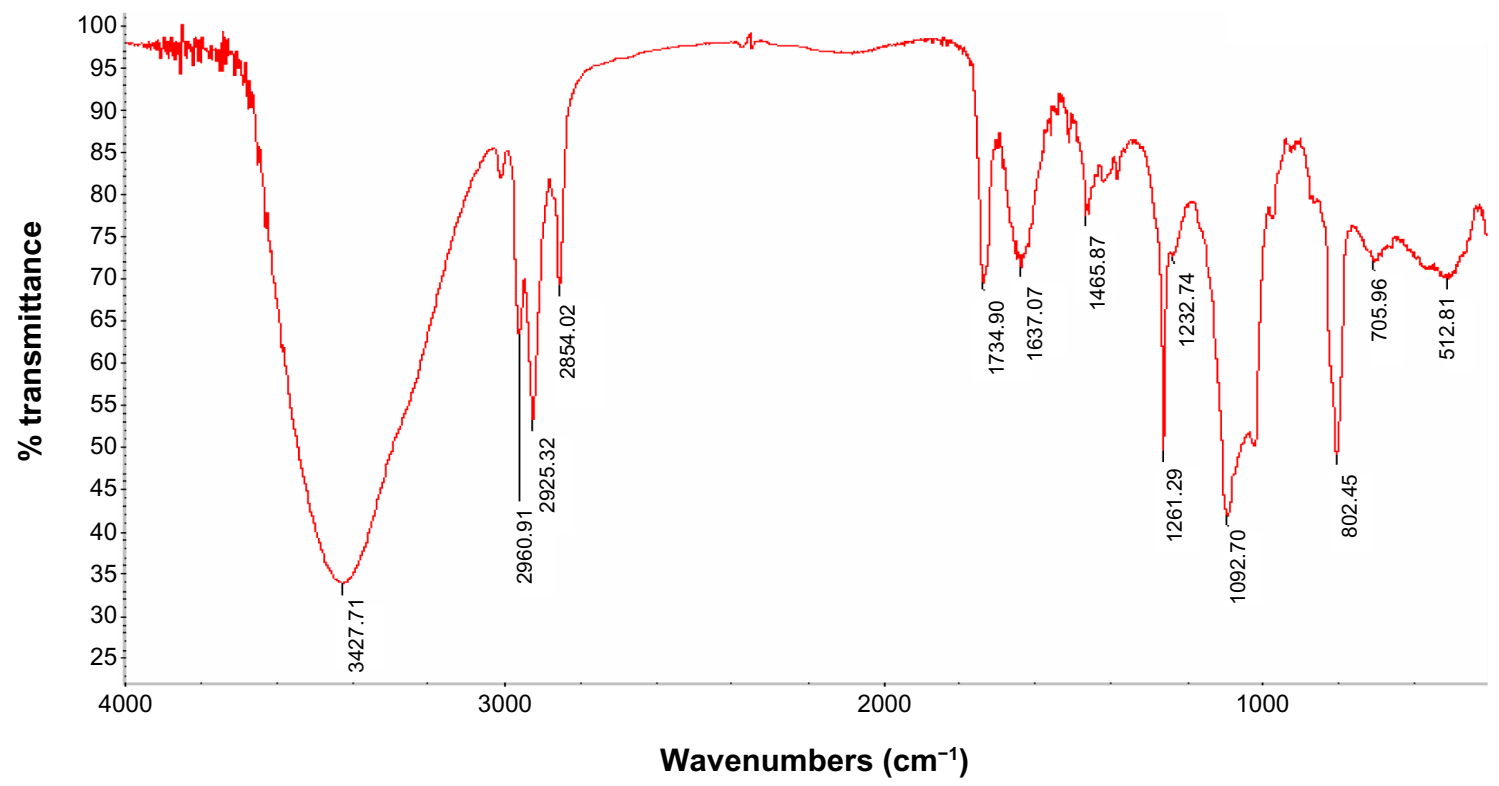

Figure 3 Fourier transform infrared spectra of $(\mathbf{A})$ tetrandrine, (B) phospholipids, (C) the physical mixture of tetrandrine and phospholipids, and (D) TPC. Abbreviation: TPC, tetrandrine-phospholipid complex.

of the individual spectra for tetrandrine and phospholipids. The tetrandrine spectrum showed characteristic peaks at $3,448.05 \mathrm{~cm}^{-1}$ and $1,163.36 \mathrm{~cm}^{-1}$. The phospholipid spectrum presented several characteristic peaks, such as the broad peak at $3,415.90 \mathrm{~cm}^{-1}$ and sharp peaks at $2,925.01 \mathrm{~cm}^{-1}$, $2,853.72 \mathrm{~cm}^{-1}$, and lower than $2,000 \mathrm{~cm}^{-1}$.

For the spectra of the TPC, the characteristic peak of tetrandrine at $1,163.36 \mathrm{~cm}^{-1}$ (the stretching vibration absorption of $\mathrm{C}-\mathrm{O}$ ) disappeared in the spectrum of the TPC complex. In addition, the peak of the polar head of phospholipids at $1,647.36 \mathrm{~cm}^{-1}(-\mathrm{C}=\mathrm{O})$ shifted to $1,637.07 \mathrm{~cm}^{-1}$ and
$1,234.55 \mathrm{~cm}^{-1}$ shifted to $1,232.74 \mathrm{~cm}^{-1}(-\mathrm{P}=\mathrm{O})$, while no significant change was found for the nonpolar side of phospholipids $\left(2,925.01 \mathrm{~cm}^{-1}\right.$ and $2,853.72 \mathrm{~cm}^{-1}$ for the $\mathrm{CH}$ structure). Therefore, it was deduced that the interaction between the $\mathrm{C}-\mathrm{O}$ of tetrandrine and the polar side of phospholipids may be responsible for complex formation by a secondary bond, van der Waals forces, and other actions. After the interaction of tetrandrine with phospholipids by polar side, the nonpolar side may move freely and enclose the polar side. The IR spectra revealed that the basic chemical structure of tetrandrine in complex was not markedly 
changed, and no new compound was formed. Furthermore, it was not a physical mixture of tetrandrine and phospholipids. Tetrandrine and phospholipids may exist in a state of interaction by intermolecular forces. ${ }^{32,33}$

\section{Preparation and characterization of TPC-LNCs}

In order to obtain the optimal formulation, an experimental design method, ${ }^{34}$ which was a central composite design, was employed with two variables (oil percentage and surfactant percentage) and two responses (droplet size and encapsulation efficiency). The data for the formulation screening and optimization are not shown here. The obtained formulation composition was Labrafil M1944CS (2.8 g), Solutol HS15 (1.9 g), phospholipid (84 mg), TPC (70 mg), deionized water (5.3 g), and $\mathrm{NaCl}(0.2 \mathrm{~g})$. The TPC-LNCs were characterized by droplet size, polydispersity measurement, encapsulation efficiency determinations, and TEM observations. The TPCLNCs had an average size of $40.0 \mathrm{~nm}$ with a polydispersity index of 0.155 . The small droplet size with a large interfacial surface area is advantageous for intestinal absorption and transport, as has been illustrated previously. ${ }^{16,35}$ Encapsulation efficiency is also an important parameter that is used to evaluate nanocarriers because a higher encapsulation may improve therapeutic effects and lower the dose of drug required. ${ }^{36,37}$ The TPC was efficiently encapsulated in LNCs, and an encapsulation efficiency as high as $93.9 \%$ was achieved. The factors influencing the encapsulation efficiency include the encapsulation process, the affinity of the drug with the carrier, and the nature of other formulation compositions. The high encapsulation that was found in this study may have been due to the formation of a drug-phospholipid complex, which enhanced the lipophilicity of tetrandrine, as was shown in the solubility study and in the affinity of tetrandrine with LNCs.

The morphological characterization of TPC-LNCs was conducted by TEM in order to obtain more information about droplet size and shape. The image is shown in Figure 4. Spherical-shaped droplets were observed.

\section{Preparation and evaluation of lyophilized TPC-LNCs}

In our preliminary study, the prepared TPC-LNCs were only stable for approximately 1 month at $37^{\circ} \mathrm{C}$, which was similar to a previously reported result. ${ }^{13}$ The presence of water may accelerate the degradation of lipids in formulation. ${ }^{38}$ Therefore, the freeze-drying process was performed in this study in order to ensure high stability and ease in storage

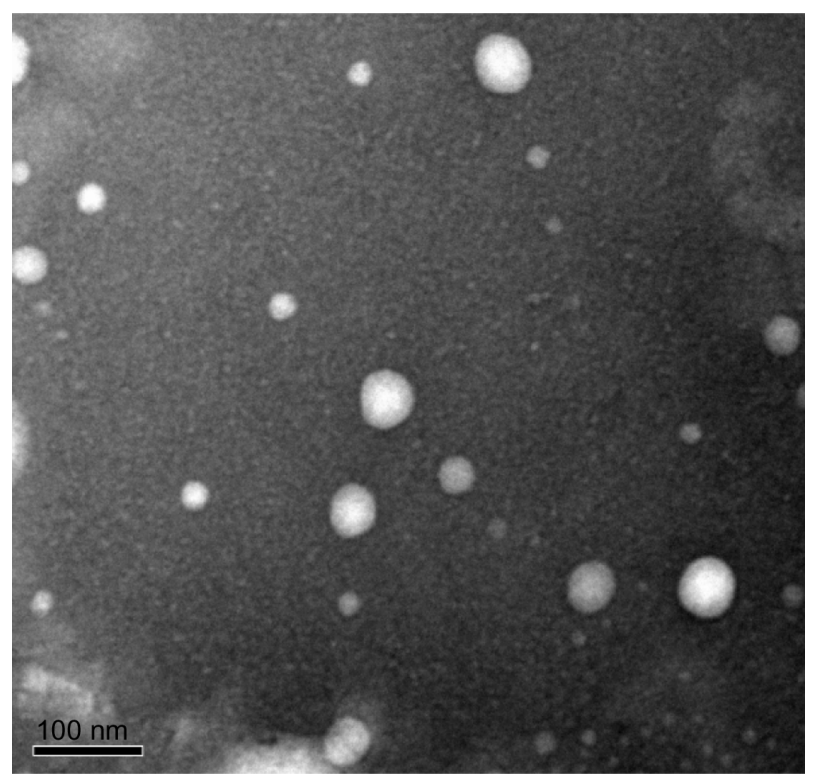

Figure 4 Transmission electron microscopic photo of TPC loaded lipid nanocapsules $(\times 100,000)$.

Abbreviation: TPC, tetrandrine-phospholipid complex.

and handling. Each cryoprotectant was added to the LNCs in the predetermined concentration. The redispersibility, including the redispersion time, the droplet size, and the polydispersity after redispersion, were used to screen the cryoprotectants. The lyophilized sample with lactose as the cryoprotectant was not capable of quickly dispersing in 30 seconds, and it was not able to attain its initial state of appearance before freeze-drying. Samples with other cryoprotectants were quickly redispersed, and their effects on droplet size and polydispersity were evaluated. These results are shown in Figure 5.

Figure 5 shows that the droplet size of lyophilized LNCs was larger compared to that before lyophilization of $40.0 \mathrm{~nm}$. The effect of the preservation of the droplets was not correlated with the concentration of the cryoprotectant. For the concentrations of $2 \%$ and $5 \%$, no significant differences were found in droplet size (variation from $125 \mathrm{~nm}$ to $135 \mathrm{~nm}$ ) after redispersion, except that of $2 \%$ glucose. Increasing the concentration of cryoprotectant to $10 \%$ improved the appearance and redispersion for all cryoprotectants. Combining the results of droplet size, redispersion time, and the appearance of the lyophilized product, mannitol (10\%) was selected as the cryoprotectant because of its loose and smooth appearance and fast redispersion with a droplet size of $145.0 \mathrm{~nm}$.

\section{DSC}

TPC-LNCs were further characterized by a DSC analysis in order to investigate the influence of TPC entrapment on the 


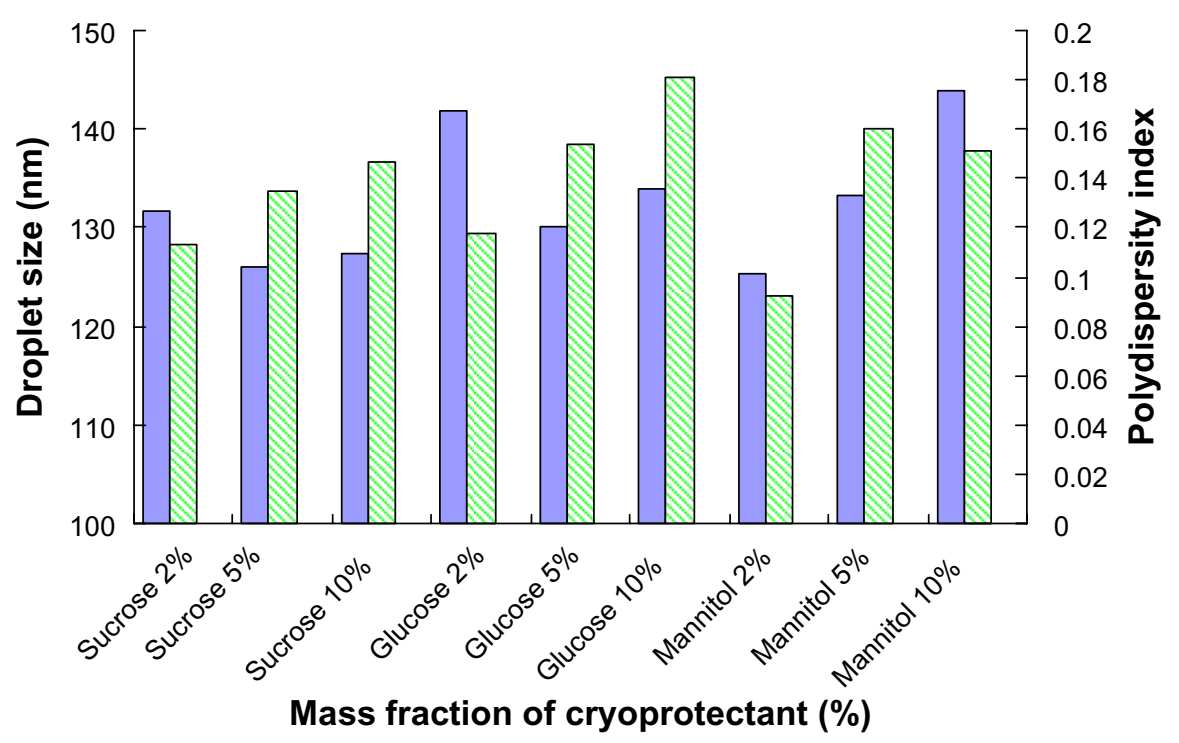

Figure 5 Effect of cryoprotectants on droplet size $(\square)$ and polydispersity index (司).

physical state of LNCs. Figure 6 shows the DSC thermograms of tetrandrine, blank LNCs, the physical mixture of tetrandrine and LNCs, and TPC-LNCs.

Tetrandrine showed one sharp peak with an onset of $225.7^{\circ} \mathrm{C}$. The physical mixture of tetrandrine and LNCs showed the same peak as tetrandrine. In the case of TPCLNCs, no tetrandrine peak appeared, indicating that tetrandrine existed in LNCs in a noncrystal state.

\section{In vitro release}

The cumulative release profile of tetrandrine free drugs, from TPC and from TPC-LNCs is shown in Figure 7.
Free tetrandrine showed a rapid release behavior, more than $30 \%$ of tetrandrine was released in the first hour, and approximately $78 \%$ was released in 4 hours. Compared to tetrandrine free drugs, the release rate of tetrandrine from TPC was slower, and approximately $40 \%$ was released in 4 hours. With respect to TPC-LNCs, more than $20 \%$ of the tetrandrine was released in the first hour. The initial first release was possibly due to the part of tetrandrine that was located at or near the interface of the nanocapsules, which may facilitate drug release from the LNCs. In addition, the small particle size may have contributed to the faster drug release as well. In the following hours,

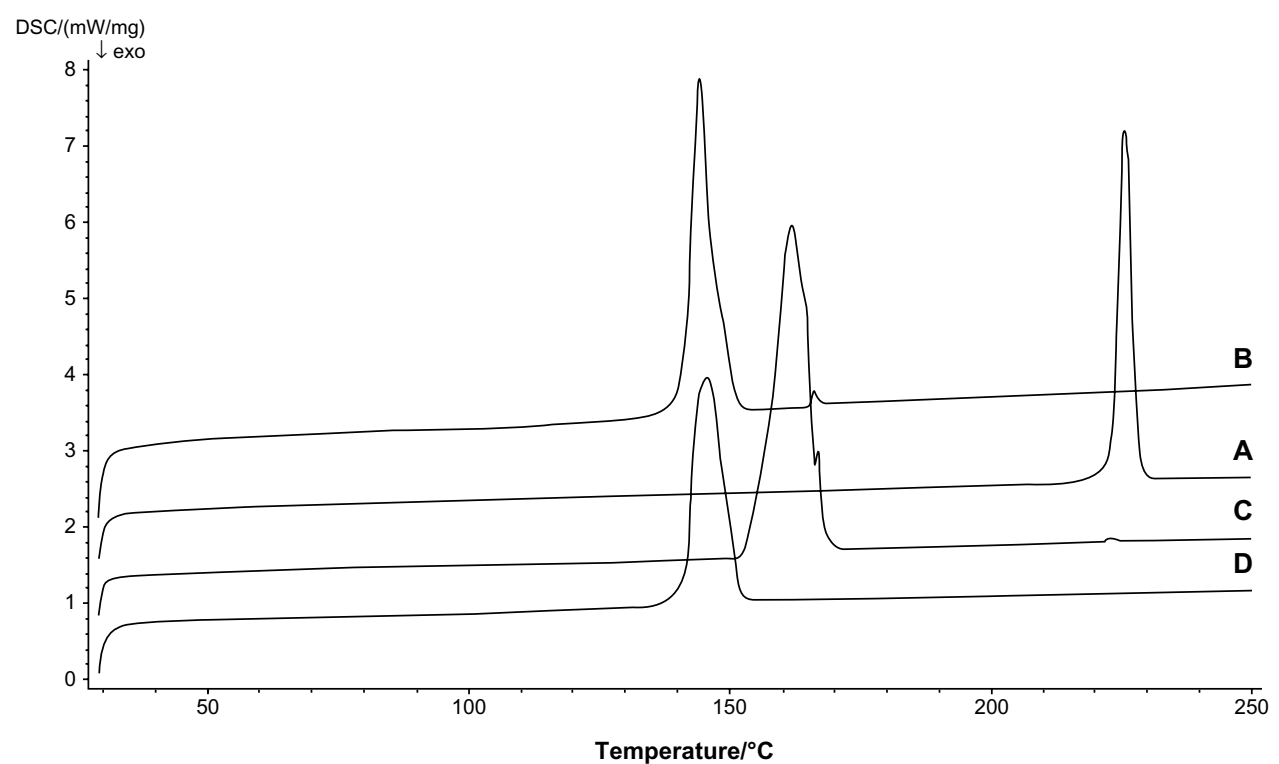

Figure 6 Differential scanning calorimetry curves for (A) tetrandrine (B) blank LNCs; (C) the physical mixture of tetrandrine and LNCs; and (D) TPC-LNCs. Abbreviations: LNCs, lipid nanocapsules; TPC-LNCs, tetrandrine-phospholipid complex loaded lipid nanocapsules; mW, milliwatt; exo, exothermal; DSC, differential scanning calorimetry. 

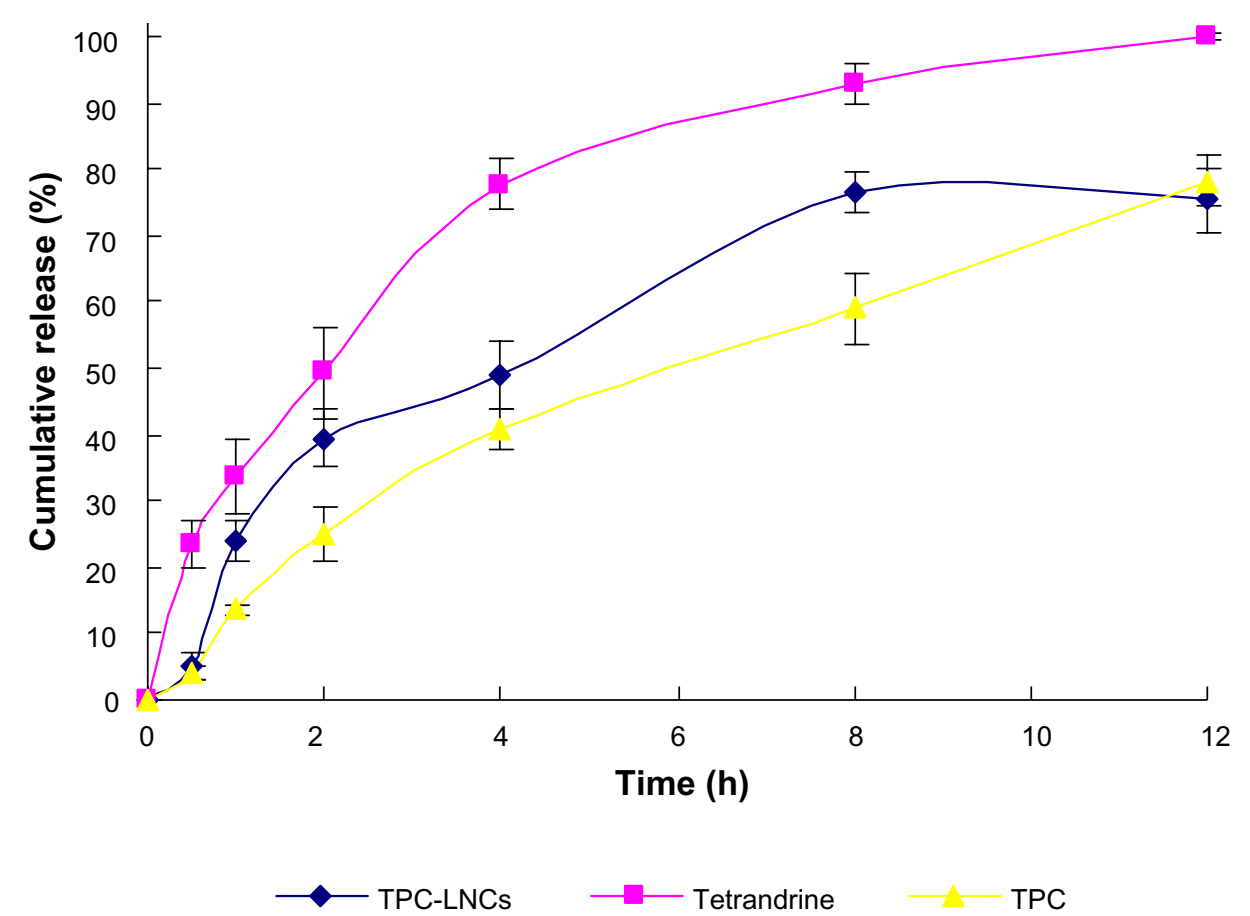

Figure $7 \mathrm{In}$ vitro release profile of tetrandrine from free tetrandrine, TPC, and TPC-LNCs $(n=3)$.

Abbreviations: h, hours; TPC, tetrandrine-phospholipid complex; TPC-LNCs, tetrandrine-phospholipid complex loaded lipid nanocapsules.

the release rate slowed, and approximately $80 \%$ of the tetrandrine was released in 8 hours. This may be attributed to the increased hydrophobicity of tetrandrine by the formation of the complex, which results in tetrandrine molecules interacting in the lipophilic domain of LNCs and the slow diffusion of tetrandrine into aqueous media..$^{35}$ Furthermore, the release rate of tetrandrine from TPCLNCs was faster than from TPC. This may be due to the high dispersibility of TPC-LNCs, which overcame the slower dissolution of TPC.

\section{In vivo bioavailability study}

The in vivo pharmacokinetic behavior of tetrandrine with LNCs and tablets was studied and compared. The plasma concentration-time profiles of tetrandrine in rats following the oral administration of TPC-LNCs and tetrandrine tablets

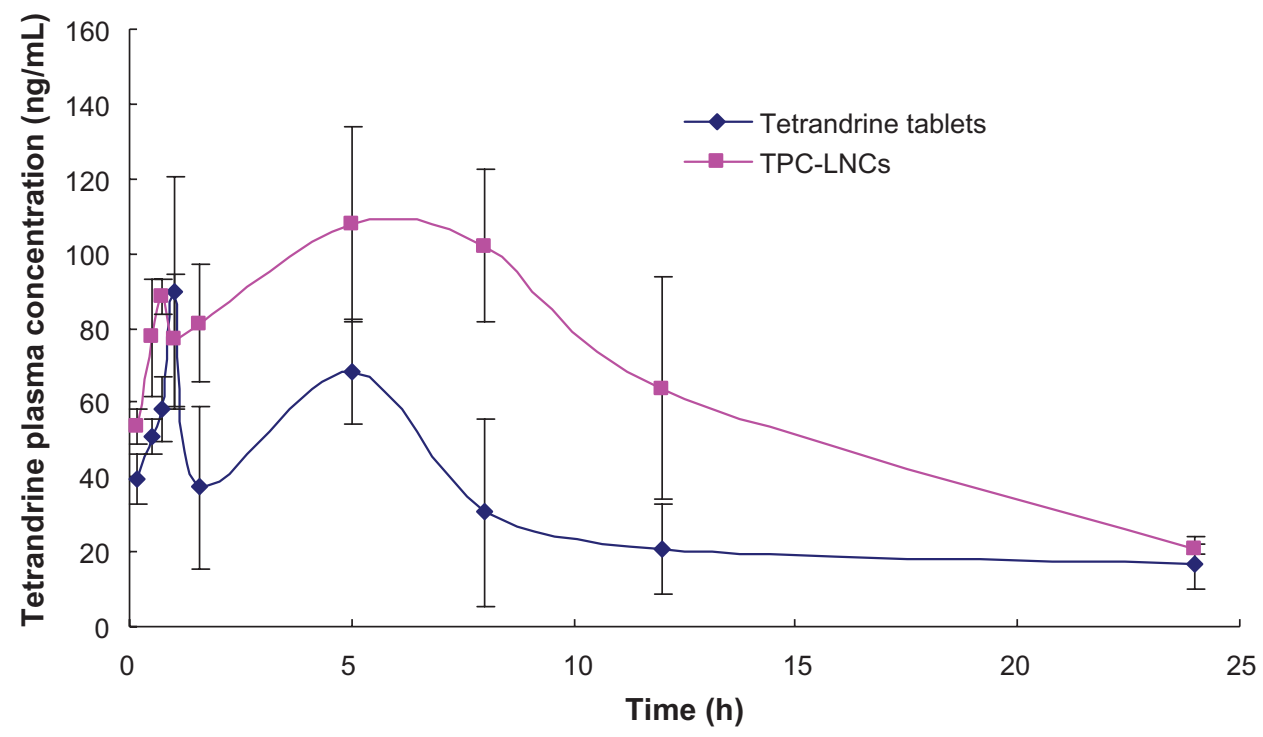

Figure 8 Plasma concentration profile of tetrandrine after the oral administration of tablets and TPC-LNCs in rats $(n=5)$. Abbreviations: h, hours; TPC-LNCs, tetrandrine-phospholipid complex loaded lipid nanocapsules. 
are shown in Figure 8. Both preparations showed two peaks in Figure 8, which was possibly caused by enterohepatic circulation.

The administration of TPC-LNCs resulted in a significant improvement in drug absorption compared to that of tablets. The relevant mean pharmacokinetic parameters are shown in Table 3.

There was a significant difference between LNCs and tablets in the AUC, which were 1,540.2 $\mu \mathrm{g} \cdot \mathrm{h} / \mathrm{L}$ and $740.5 \mu \mathrm{g} \cdot \mathrm{h} / \mathrm{L}$, respectively $(P<0.05)$. No significant difference was observed in $\mathrm{T}_{\max }$ and $\mathrm{C}_{\max }$. The relative bioavailability of the LNCs was approximately 2.1-fold compared to the tablets, indicating that the bioavailability of tetrandrine was significantly improved by LNCs.

There are several possible reasons for the improvement. First, phospholipids may play an important role in drug absorption. After the formation of the TPC, the lipophilicity of tetrandrine was significantly increased, which may facilitate the absorption of tetrandrine into the intestinal mucosa. Moreover, phospholipids, which are an important component of the cell membrane, may improve the drug passage across the lipid-rich biological membranes. ${ }^{39,40}$ In addition, the lipidic nature of the nanocapsules may have contributed to the improvement of bioavailability to similar levels as other lipid formulations. From this aspect, the possible mechanisms include the presence of lipids and surfactants that facilitate the dissolving of the drug and dissolution, improved drug permeability by enhancing uptake through various routes or by inhibiting the efflux of drugs due to some excipient in formulation, and/or absorption through the intestinal lymphatic transport system. ${ }^{16}$ Another possible reason is that, upon entering the gastrointestinal tract, the formation of numerous liquid droplets of LNCs that are nanosized could greatly expand the contact area with the gastrointestinal tract. The

Table 3 The main pharmacokinetic parameters after the oral administration of TPC-LNCs or tetrandrine tablets in rats $(n=5)$ at a dose of $5.4 \mathrm{mg} \cdot \mathrm{kg}^{-1}$

\begin{tabular}{lll}
\hline Parameters & Tablets & TPC-LNCs \\
\hline $\mathrm{AUC}_{0-\mathrm{t}}(\mu \mathrm{g} \cdot \mathrm{h} / \mathrm{mL})$ & $0.74 \pm 0.034$ & $1.54 \pm 0.452^{* *}$ \\
$\mathrm{AUC}_{0-\infty}(\mu \mathrm{g} \cdot \mathrm{h} / \mathrm{mL})$ & $1.83 \pm 0.062$ & $1.98 \pm 0.246$ \\
$\mathrm{MRT}_{0-\mathrm{t}}(\mathrm{h})$ & $7.58 \pm 3.268$ & $8.39 \pm 1.532$ \\
$\mathrm{~T}_{\text {max }}(\mathrm{h})$ & $1.92 \pm 1.74 \mathrm{I}$ & $4.92 \pm 2.265$ \\
$\mathrm{C}_{\text {max }}(\mu \mathrm{g} / \mathrm{mL})$ & $0.096 \pm 0.024$ & $0.116 \pm 0.020$ \\
Relative bioavailability $(\%)$ & - & 207.98 \\
\hline
\end{tabular}

Notes: The data are expressed as mean \pm standard deviation $(n=5)$. $* * P<0.01$ is the statistical significance of the comparison of tablets versus LNCs.

Abbreviations: AUC, area under the curve; $C_{\max }$, peak concentration; h, hours; MRT, mean residence time; $T_{\max }$, peak time; TPC-LNCs, tetrandrine-phospholipid complex loaded lipid nanocapsules. exact absorption mechanism of phospholipids in this study will be investigated in our future work.

It is worth noting that the number of blood taking may be of concern when studying drug-phospholipid complex loaded LNCs. An excess of blood taking in rats may result in the decreased blood flow rate. Larger animals, such as rabbits or dogs, may be more preferable models. The influence of the animal model on the bioavailability of LNCs still needs further investigation.

\section{Conclusion}

The current study showed the potential use of a drugphospholipid complex for the preparation of LNCs in order to enhance the oral bioavailability of a drug with moderate lipophilicity. TPC-LNCs that were produced by the phase inversion method were spherical-shaped particles with a small size. High encapsulation efficiency was achieved due to the formation of TPC. TPC-LNCs were lyophilized with mannitol (10\%) as a cryoprotectant exhibited a loose and smooth appearance, and fast redispersion. The in vitro release study showed fast release in the first hour which was followed by a sustained release over 12 hours. In addition, the TPC-LNCs significantly improved the bioavailability of tetrandrine compared to that of the tablets. These results suggested that formulating TPC into LNCs may efficiently enhance the encapsulation efficiency of the moderately lipophilic drug and may further improve its oral bioavailability.

\section{Acknowledgments}

This work was financially supported by grant 2010 JW12 from the Shanghai Education Committee, Program 11ZR1436500 from the Science and Technology Commission of Shanghai Municipality, the National Natural Science Foundation of China (No 81202925), and the Specialized Research Fund for the Doctoral Program of Higher Education of China (20123107110005).

\section{Disclosure}

The authors report no conflicts of interest in this work.

\section{References}

1. Cai XH, Wang S, Chen BA. Research advances on the pharmacological effects of tetrandrine. Chin J Nat Med. 2011;6:473-480.

2. Wu JM, Chen Y, Chen JC, Lin TY, Tseng SH. Tetrandrine induces apoptosis and growth suppression of colon cancer cells in mice. Cancer Lett. 2010;287(2):187-195.

3. Chen B, Yin L, Cheng J, et al. Effect of D, L-threo-1-phenyl-2decanoylamino-3-morpholino-1-propanol and tetrandrine on the reversion of multidrug resistance in K562/A02 cells. Hematology. 2011;16(1):24-30.

4. Cai XH, Wang S, Chen BA. Research advances on the pharmacological effects of tetrandrine. Chin J Nat Med. 2011;9(6):473-480. 
5. Takemura H, Imoto $\mathrm{K}$, Ohshika $\mathrm{H}, \mathrm{Kwan} \mathrm{CY}$. Tetrandrine as a calcium antagonist. Clin Exp Pharmacol Physiol. 1996;23(8):751-753.

6. Tian X, Zhu JB, Wang JS, Li TF. Preparation of tetrandrine lipid emulsion and its therapeutic effect on bleomycin-induced pulmonary fibrosis in rats. Journal of China Pharmaceutical University. 2005; 36(3):225-229.

7. Li S, Ji Z, Zou M, Nie X, Shi Y, Cheng G. Preparation, characterization, pharmacokinetics and tissue distribution of solid lipid nanoparticles loaded with tetrandrine. AAPS PharmSciTech. 2011;12(3): 1011-1018.

8. Chen HW, Wang YL, You GY, Chen ZP. Study on pharmacokinetics of tetrandrine liposomes in rats. Journal of Nanjing TCM University. 2010;26(5):353-355.

9. Zeng XM, Martin GP, Marriott C. Tetrandrine delivery to the lung: The optimisation of albumin microsphere preparation by central composite design. Intl J Pharm. 1994;109(2):135-145.

10. Desai PP, Date AA, Patravale VB. Overcoming poor oral bioavailability using nanoparticle formulations-opportunities and limitations. Drug Discov Today. 2012;9(2):e87-e95.

11. Chakraborty S, Shukla D, Mishra B, Singh S. Lipid - an emerging platform for oral delivery of drugs with poor bioavailability. Eur $J$ Pharm Biopharm. 2009;73(1):1-15.

12. Huynh NT, Passirani C, Saulnier P, Benoit JP. Lipid nanocapsules: a new platform for nanomedicine. Int J Pharm. 2009;379(2):201-209.

13. Heurtault B, Saulnier P, Pech B, Proust JE, Benoit JP. A novel phase inversion-based process for the preparation of lipid nanocarriers. Pharm Res. 2002;19(6):875-880.

14. Hureaux J, Lagarce F, Gagnadoux F, et al. Lipid nanocapsules: readyto-use nanovectors for the aerosol delivery of paclitaxel. Eur J Pharm Biopharm. 2009;73(2):239-246.

15. Sánchez-Moreno P, Ortega-Vinuesa JL, Martín-Rodríguez A, Boulaiz H, Marchal-Corrales JA, Peula-García JM. Characterization of different functionalized lipidic nanocapsules as potential drug carriers. Int J Mol Sci. 2012;13(2):2405-2424.

16. Peltier S, Oger JM, Lagarce F, Couet W, Benoît JP. Enhanced oral paclitaxel bioavailability after administration of paclitaxel-loaded lipid nanocapsules. Pharm Res. 2006;23(6):1243-1250.

17. Khalid MN, Simard P, Hoarau D, Dragomir A, Leroux JC. Long circulating poly(ethylene glycol)-decorated lipid nanocapsules deliver docetaxel to solid tumors. Pharm Res. 2006;23(4):752-758.

18. Liu Y, Pan J, Feng SS. Nanoparticles of lipid monolayer shell and biodegradable polymer core for controlled release of paclitaxel: effects of surfactants on particles size, characteristics and in vitro performance. Int J Pharm. 2010;395(1-2):243-250.

19. Thanki K, Gangwal RP, Sangamwar AT, Jain S. Oral delivery of anticancer drugs: challenges and opportunities. $J$ Control Release. 2013;170(1):15-40.

20. Kohli K, Chopra S, Dhar D, Arora S, Khar RK. Self-emulsifying drug delivery systems: an approach to enhance oral bioavailability. Drug Discov Today. 2010;15(21-22):958-965.

21. Peng Q, Zhang ZR, Sun X, Zuo J, Zhao D, Gong T. Mechanisms of phospholipid complex loaded nanoparticles enhancing the oral bioavailability. Mol Pharm. 2010;7(2):565-575.

22. Yue PF, Yuan HL, Li XY, Yang M, Zhu WF. Process optimization, characterization and evaluation in vivo of oxymatrine-phospholipid complex. Int J Pharm. 2010;387(1-2):139-146.
23. Wu J, Chen D, Zhang R. Study on the bioavailability of baicalinphospholipid complex by using HPLC. Biomed Chromatogr. 1999; 13(7):493-495.

24. Xia HJ, Zhang ZH, Jin X, Hu Q, Chen XY, Jia XB. A novel drugphospholipid complex enriched with micelles: preparation and evaluation in vitro and in vivo. Int J Nanomedicine. 2013;8:545-554.

25. Cui F, Shi K, Zhang L, Tao A, Kawashima Y. Biodegradable nanoparticles loaded with insulin-phospholipid complex for oral delivery: preparation, in vitro characterization and in vivo evaluation. $J$ Control Release. 2006;114(2):242-250.

26. Ruan J, Liu J, Zhu D, et al. Preparation and evaluation of selfnanoemulsified drug delivery systems (SNEDDSs) of matrine based on drug-phospholipid complex technique. Int J Pharm. 2010;386(1-2): 282-290.

27. Peng Q, Zhang ZR, Gong T, Chen GQ, Sun X. A rapid-acting, longacting insulin formulation based on a phospholipid complex loaded PHBHHx nanoparticles. Biomaterials. 2012;33(5):1583-1588.

28. Liu Y, Wang P, Sun C, et al. Wheat germ agglutinin-grafted lipid nanoparticles: preparation and in vitro evaluation of the association with Caco-2 monolayers. Int J Pharm. 2010;397(1-2):155-163.

29. Semalty A, Semalty M, Singh D, Rawat MSM. Preparation and characterization of phospholipid complexes of naringenin for effective drug delivery. J Incl Phenom Macrocycl Chem. 2010;67(3-4):253-260.

30. Bunjes H, Unruh T. Characterization of lipid nanoparticles by differential scanning calorimetry, X-ray and neutron scattering. Adv Drug Deliv Rev. 2007;59(6):379-402.

31. Fricker G, Kromp T, Wendel A, et al. Phospholipids and lipid-based formulations in oral drug delivery. Pharm Res. 2010;27(8):1469-1486.

32. Tan Q, Liu S, Chen X, et al. Design and evaluation of a novel evodiamine-phospholipid complex for improved oral bioavailability. AAPS PharmSciTech. 2012;13(2):534-547.

33. Maiti K, Mukherjee K, Gantait A, Saha BP, Mukherjee PK. Curcuminphospholipid complex: Preparation, therapeutic evaluation and pharmacokinetic study in rats. Int J Pharm. 2007;330(1-2):155-163.

34. Gil EC, Colarte AI, Bataille B, Pedraz JL, Rodríguez F, Heinämäki J. Development and optimization of a novel sustained-release dextran tablet formulation for propranolol hydrochloride. Int $J$ Pharm. 2006;317(1):32-39.

35. Lamprecht A, Bouligand Y, Benoit JP. New lipid nanocapsules exhibit sustained release properties for amiodarone. J Control Release. 2002;84(1-2):59-68.

36. Lamprecht A, Saumet JL, Roux J, Benoit JP. Lipid nanocarriers as drug delivery system for ibuprofen in pain treatment. Int $J$ Pharm. 2004;278(2):407-414.

37. Cheng X, Liu R, He Y. A simple method for the preparation of monodisperse protein-loaded microspheres with high encapsulation efficiencies. Eur J Pharm Biopharm. 2010;76(3):336-341.

38. Varshosaz J, Eskandari S, Kennedy R, Tabbakhian M, Minaiyan M. Factors affecting the production of nanostructure lipid carriers of valproic acid. J Biomed Nanotechnol. 2013;9(2):202-212.

39. Cai X, Luan Y, Jiang Y, et al. Huperzine A-phospholipid complexloaded biodegradable thermosensitive polymer gel for controlled drug release. Int J Pharm. 2012;433(1-2):102-111.

40. Li J, Liu P, Liu JP, et al. Bioavailability and foam cells permeability enhancement of Salvianolic acid B pellets based on drug-phospholipids complex technique. Eur J Pharm Biopharm. 2013;83(1):76-86.
International Journal of Nanomedicine

\section{Publish your work in this journal}

The International Journal of Nanomedicine is an international, peerreviewed journal focusing on the application of nanotechnology in diagnostics, therapeutics, and drug delivery systems throughou the biomedical field. This journal is indexed on PubMed Central, MedLine, CAS, SciSearch ${ }^{\circledR}$, Current Contents ${ }^{\circledR} /$ Clinical Medicine,

\section{Dovepress}

Journal Citation Reports/Science Edition, EMBase, Scopus and the Elsevier Bibliographic databases. The manuscript management system is completely online and includes a very quick and fair peer-review system, which is all easy to use. Visit http://www.dovepress.com/ testimonials.php to read real quotes from published authors. 